\title{
STRUCTURAL AND BIOMECHANICAL PROPERTIES OF A NOVEL 3D MICRODERMIS MODEL: THE SPHEROID
}

Chloé Lorion PhD ${ }^{1}$, Amandine Lopez-Gaydon' ${ }^{1}$, Sébastien Bonnet ${ }^{1}$, Anna Drillat ${ }^{2}$,

Pascale Milani ${ }^{2} \mathrm{PhD}$, Nicolas Bechetoille ${ }^{1} \mathrm{PhD}$

${ }^{1}$ Gattefossé - 36 chemin de Genas - CS 70070 - 69804 SAINT-PRIEST Cedex - France; ${ }^{2}$ BioMeca SAS, 46 allée d'Italie, Lyon, France

\section{INTRODUCTION}

Spheroids as microtissues are a powerful alternative to standard 2D cell culture for in vitro studies. 3D scaffold-free spheroids are formed within a few days from a cell suspension that aggregates in Ultra Low Affinity plates (ULA plates). The advantages of spheroids exclusively composed of fibroblasts rely on the physiological production of the extracellular matrix thanks to the aggregative capability of fibroblasts to self-assemble in a round tridimensional structure. This microdermis presents a complex tissue organization that closely mimics the architecture and composition of the human dermis in vivo. The aim of this study was to characterize structural and biomechanical properties of spheroids composed of normal human fibroblasts at 8 and 15 days of culture.

\section{MATERIALS \& METHODS}

\section{D Microdermis formation}

GravityTRAP ULA plate (Microtissue Culture and Assay Plateform, PerkinElmer)

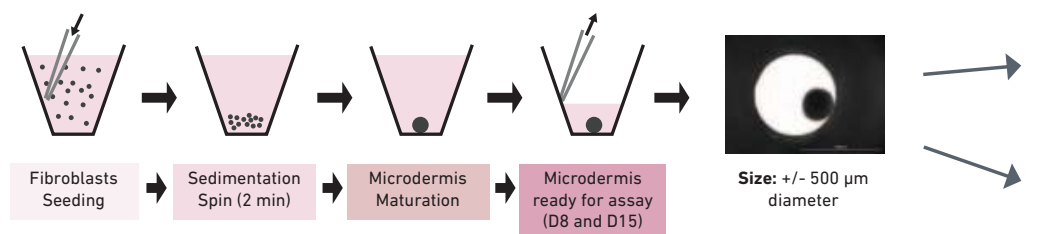

\section{Biomechanical characterization}

AFM and SHG analyzes at D8 and D15

(1) Elastic fibers density

Analysis method: SHG on spheroids

(2) Biomechanical properties

Analysis method: AFM on spheroids
(3) Correlation between

(1) \& (2)

Analysis method:

Immunofluorescence and

AFM on spheroids sections

\section{RESULTS}

(1) Elastic fibers density within the microdermis

Second Harmonic Generation microscopy on spheroids at D8 and D15

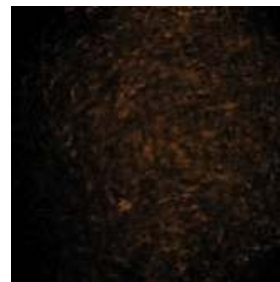

Sph. D8

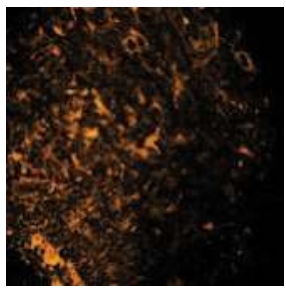

Sph. D15
Increase of elastin synthesis at D15 compared to D8

Figure 1: Z projection images of elastin deposits within fibroblast spheroids afte 8 days and 15 days of culture. Image realized by Second Harmonic Generation Microscopy (Objective X40).

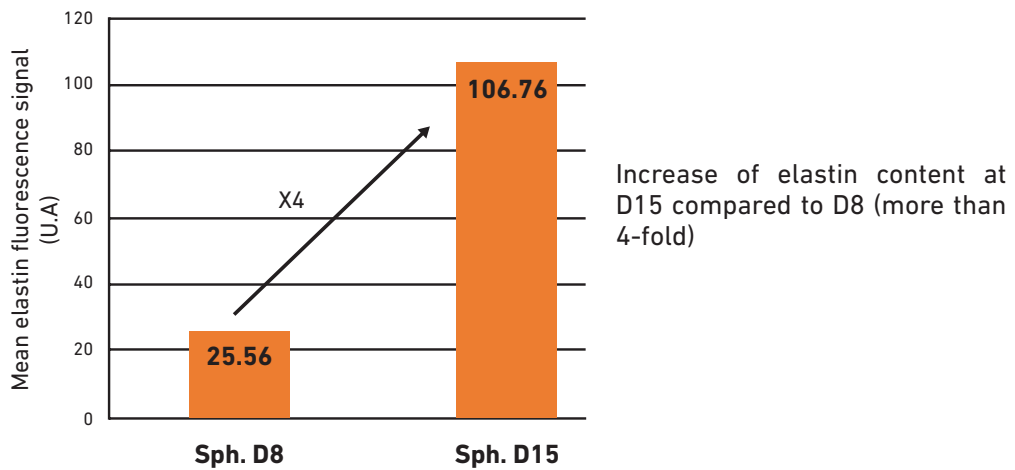

Figure 2: Analyzes of the SHG images. Mean elastin fluorescence signal quantification (grey level) within fibroblast spheroids after 8 days and 15 days of culture.

\section{(2) Biomechanical properties of the microdermis}

Atomic Force Microscopy on spheroids at D8 and D15

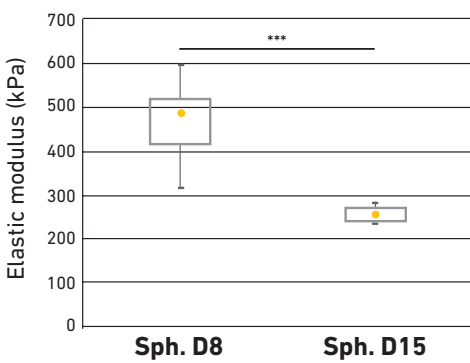

Decrease of elastic modulus at D15 compared to D8

Decrease of apparent stiffness of the microtissue at D15 compared to D8

Figure 3: Distribution of elastic modulus extracted from fibroblast spheroids at 8 days and 15 days of culture. Student test: ${ }^{* * *} p$-value $<0,0005$

\section{(3) Correlation between elastic modulus} maps and elastin deposits density

Immunofluorescence and AFM on spheroids sections at D15
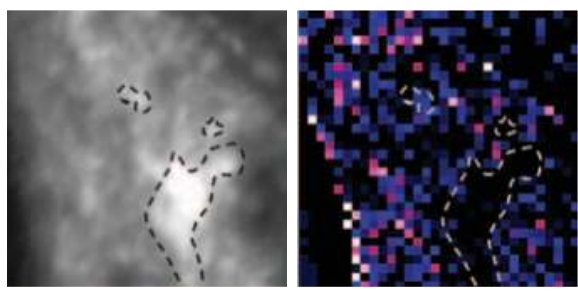
$600 \mathrm{kPa}$

Areas rich in elastin within fibroblat spheroids correspond to less stiffness areas (lower elastic modulus)

Figure 4: Immunofluorescence imaging of elastin within fibroblast spheroids at D15. Each image is associated with its mechanical properties map (elastic modulus is represented in $\mathrm{kPa}$ ). Areas rich in elastin are identified by dots and correspond to soft areas.

\section{CONCLUSION}

These results highlight the elastic properties of the microdermis at D15, which is mechanically softer than a spheroid at D8, and this thanks to the presence of elastic fibers. Therefore, a spheroid at D15 is a biomimetic model in which mechanical and structural elastic properties are related to each other. This correlative study outlines spheroids as a reliable microdermis model to test active ingredient efficiency on elastin synthesis and biomechanical properties of the microtissue.
Take-home message
(1) Microdermis at D15 is rich in elastic fibers

Microdermis at D15 is than at D8
(3) microdermis at D15 are mechanically softer 